\title{
Austrotransplant 2007 Österreichisch-deutsche Zusammenarbeit angebahnt
}

Im Oktober 2007 tagte der Österreichische Pflegeverein für Transplantationschirurgie am Wolfgangsee. Neben den Vorträgen von Pflegekräften bot sich die Möglichkeit zum kollegialen Austausch. Der lange Zeit ruhende Kontakt zwischen deutschen und österreichischen Transplantationspflegekräften wurde wieder aufgenommen und ein stärkerer Austausch für die Zukunft geplant.

Unter dem Motto „Ganz jung - Ganz alt“ fand die 15. ÖPTC-Pflegetagung (Österreichischer Pflegeverein für Transplantationschirurgie) vom 17.-19. Oktober vergangenen Jahres in St. Wolfgang am Wolfgangsee statt. Da es in ganz Österreich nur vier Transplantationszentren gibt, in denen Nieren verpflanzt werden (Wien, Graz, Innsbruck und Linz), war der Charakter der Veranstaltung fast als familiär zu bezeichnen.

Am Begrüßungsabend gab es erste Gelegenheit zum kollegialen Erfahrungsaustausch. So konnten Pflegekräfte aus Graz helfen, den Kontakt zwischen ÖPTC und AKTX wiederherzustellen. Herbert Janisch (Obmann ÖPTC) und Richard
Weisz (stellvertretender Obmann ÖPTC) hatten vor Jahren in Köln das erste AKTX-Pflegesymposium besucht, danach sei die Verbindung jedoch eingeschlafen. Umso mehr freuten sie sich über die Kontaktaufnahme von Seiten der AKTX und hoffen auf einen regen Austausch auch in Zukunft über die nationalen Grenzen hinweg.

\section{Beitrag zur Pflegeproblematik bei} der Kinderdialyse ausgezeichnet Am nächsten Morgen eröffnete die Diplomgesundheitskrankenschwester Claudia Mayer von der Akutdialyse im AKH Wien als organisatorische Leitung das Seminar. Sämtliche Vortragenden

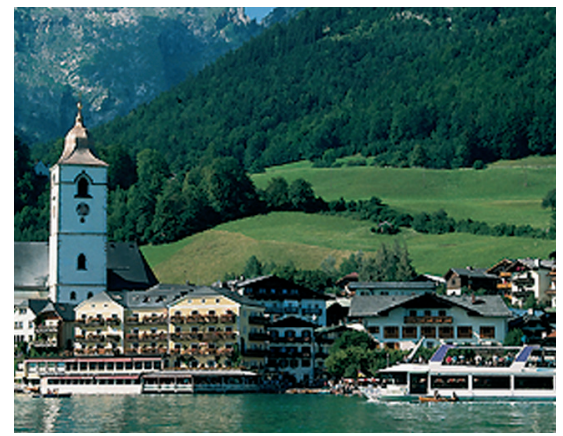

waren Pflegekräfte. Über spezielle Pflegemaßnahmen bei Transplantierten gibt es auch in Österreich unterschiedliche Meinungen, zum Beispiel über Sinn und Zweck eines Betaisadonna-Bades vor der Transplantation.

Rund 80 Pflegekräfte nahmen an der Veranstaltung teil, wobei ein relativ großer Anteil an Pflegekräften aus Dialysezentren kam. Allerdings muss in Österreich jede examinierte Pflegekraft mindestens 40 Fortbildungsstunden innerhalb von fünf Jahren nachweisen und bei einer solchen Veranstaltung ist (in schöner Umgebung) schnell ein Teil davon absolviert.

Am Freitagabend wurden im Rahmen einer Festveranstaltung die drei besten Beiträge mit einem Preis ausgezeichnet, den die Firmen KCI und Inovamet gestiftet hatten. Der erste Preis ging an Hermine Szivacz-Bösel und Gabriele Mürwald vom AKH Wien für den Beitrag „Freud und Leid an der Kinderdialyse aus Sicht der Pflege“. Den zweiten Preis erhielt Herbert Janisch vom Klinikum Graz für seinen Vortrag zur Pflege bei Patienten mit Assist-Device-Systemen. Den dritten Platz belegte Christoph Dielacher vom AKH Wien mit seinem Vortrag zum Kardiomyopathiepatienten.

Bei meiner Verabschiedung sprachen die Herren Janisch und Weisz nochmals die Hoffnung aus, den zwischen ÖPTC und AKTX geknüpften Kontakt durch weitere gemeinsame Treffen zu vertiefen.

Barbara Gnatz, München 